\title{
Mechanical oscillations in biological tissues as a result of delayed rest-length changes
}

\author{
José J. Muñoz ${ }^{1}$, Mónica Dingle ${ }^{1}$, Manuel Wenzel ${ }^{2}$
}

October 30, 2018

j.munoz@upc.edu, www.lacan.upc.edu/munoz

1. Dept. Mathematics, Universitat Politècnica de Catalunya-Barcelona Tech

2. Karslruhe Institute of Technology

\begin{abstract}
Oscillatory behaviour in tissue biology is ubiquitous and may be observed in the form of either chemical or mechanical signals. We here present and solve a mechanical cell model which exhibits oscillations emerging from delayed viscoelastic rheological laws. These include a time delay between the mechanical response and the rest-length changes, which evolve proportionally to the delayed cell deformation and use a remodelling rate parameter. We show that different regimes (no oscillatory response, sustained oscillations, and unstable oscillations) are obtained for different values of the delay or the remodelling rate.

The results are analytically demonstrated in a one-dimensional problem with one and two cells which are represented by simple line elements. Oscillations of the cell deformations are obtained whenever different delays co-exist, or the delay is size-dependent. We also extend our results to a multicellular two-dimensional vertex model that includes the same rheological law, and which inherits the presence of critical values of the delay or remodelling rate. We numerically show that indeed the size-dependent rest-length changes induce oscillations in the cell shapes and areas.
\end{abstract}

\section{Introduction}

Oscillatory responses in tissues are observed in numerous phenomena: gene regulatory signalling [1], cardiac pulsation [2], shear waves in cardiac tissue [3], chemical synapses in neural loops [4] or pattern formation during embryo segmentation $[5,6]$, to name a few. The suggested physical causes behind the observed oscillations are also varied: cardiac electrical impulses [2], cycling gene expression [6], inertial terms [3], or delays produced by distance in signalling and responses $[5,4]$. In turn, different methods have been used to model these systems: activator-repressor coupling in lattices [1], second order differential equations [3], control differential equations with feedback [2], diffusion-reaction 
equations $[7,8]$, or delayed contractile forces $[9,10]$. Despite these studies, it is yet unclear how these ubiquitous oscillatory responses are originated in in vitro [11] or in vivo epithelia [9].

In this study we concentrate our attention on displacement oscillations observed in embryonic tissues [10], and model them through a delayed response. Previous use of a delay for modelling oscillators can be found for instance in $[5,9]$, and has been justified by the presence of a distance between the sender and the receiver of the biochemical signal, or due to the time needed to process such signal. We will not investigate the causes behind this delay, but rather analyse its consequences on the oscillatory behaviour and stability of the cell deformation. We show that the delay strongly affects the stability, which in turn may help to explain embryonic development. In embryo morphogenesis, area oscillations appear precisely prior to drastic shape changes of the organism, and have been suggested as a potential mechanism originating ventral invagination [12], dorsal closure [9, 13] or symmetry breaking [14] in Drosophila fly.

The dynamics of these cell area fluctuations have a strong resemblance to wave-like oscillations of a fluid surface (see movies in the supplemental material of [9] and Figure 1 in [10], which illustrate the oscillations at the apical surfaces). However, in cellular systems, inertial terms are negligible, which renders the governing mechanical partial differential equations as elliptical or parabolic, with no apparent oscillatory solution. It is thus necessary to search for other sources that may trigger such fluctuations. Recently, these oscillations have been correlated with contraction pulses and myosin concentration pulsations $[15,16,17]$. Moreover, the time lag between mechanical displacements and concentration oscillations has been experimentally measured, and is approximately equal to one quarter of the wave period $(\sim 230 s)$ [10].

Motivated by these observations and experimental measurements, we here resort to a minimal system that includes elastic mechanical equilibrium and a delay in the cell active response. The latter is represented by an evolution equation of the cell rest-length that gives rise to a delay differential equation (DDE). DDEs have been extensively used in control systems [18] and have been recently explored in cellular oscillators [5]. In the context of embryogenesis, contractile forces with delays have been also employed in [9]. However, the stability and oscillatory conditions have not been analysed for such cellular systems.

The coupling between cortex turnover, its contractility, mechanical equilibrium and the measured oscillations have been studied for a two cell system in [19], while the associated myosin transport and cell area changes are modelled in [10]. In contrast to these works, we decide to omit the solution of transport diffusion-reaction equations. In our model, the delay in the evolution equation is attributed to the retarded bio-chemical signalling, and simulates the coupling of the myosin concentration and the cortex contractility. By directly inserting the delay between the cell mechanical state and the active response, we manage to retrieve analytical conditions for the stability as a function of this delay and the cell material properties. 


\section{Active rheological model with delay}

\section{$2.1 \quad$ Single element analysis}

We here model the cell with a line element that has a simple linear elastic law. The element is schematised in Figure 1a, and has a stress given by $\sigma=k \varepsilon^{e}$, with $\varepsilon^{e}=\ell-L$ a displacement based strain measure, and $\ell$ and $L$ the lengths at the deformed and stress free configuration. In order to mimic a viscoelastic response, we resort to the following evolution law for the rest-length $L$ [20]:

$$
\dot{L}=\gamma\left(\varepsilon^{e}(t)-\varepsilon^{c}\right) .
$$

Contractility parameter $\varepsilon^{c}$ corresponds to a material homeostatic value at which $L$ is constant [21]. It has been shown that this law, in conjunction with the linear elastic law mimics a Maxwell model with viscosity $\eta$, where the characteristic time $k / \eta$ is here given by the remodelling rate $\gamma$ [20]. Similar models based on rest-length changes have been employed for embryonic tissues in order to represent their ability to adapt and remodel $[22,23]$.

For simplicity in our subsequent analysis, we assume that $\varepsilon^{c}=0$, although the validity of the methodology and conclusions remain unchanged without this assumption. We further hypothesise that the rest-length adapts with a given delay $\tau$, so that equation (1) adopts the following expression,

$$
\dot{L}=\gamma(l(t-\tau)-L(t-\tau)) .
$$

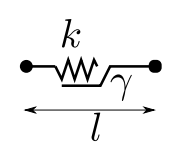

(a)

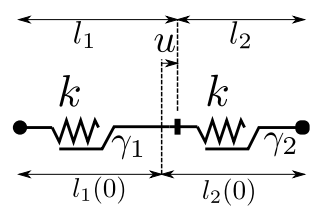

(b)

Figure 1: (a) Schematic of model for 1 cell. (b) Model with 2 cells.

We now impose a constant stretch $u=l-l_{0}$, with an initial rest-length $L(0)=L_{0}=l_{0}, \tau \leq t \leq 0$. The resulting delay differential equation (DDE) and initial conditions reads:

Find $L(t), \forall t>0$, that satisfies

$$
\dot{L}=\gamma(l-L(t-\tau))
$$

with constants $l>l_{0}, \gamma>0, \tau>0$ and initial conditions

$$
L(t)=l_{0},-\tau \leq t \leq 0 .
$$

The stability of the DDE can be analysed resorting to the solution of the characteristic equation $[18,24,25]$. It is known that there is a critical value

$\tau_{\text {oscil }}$ of the delay for which the rest-length $L(t)$ oscillates, and another value 
$\tau_{\text {stab }}$ above which the solution is unstable, giving oscillations with increasing amplitude. We will show that in our case these limiting values depend on the remodelling rate $\gamma$. We will recall the main results and methodology, which will be recalled in the analysis of the 2 element system shown in Figure 1b. .

We will assume that the solution can be written as a linear combination of exponential forms $L(t)_{m}=\left(L_{0}-l_{0}\right) e^{m t}+l_{0}$. By inserting this form in the DDE in (3), with $m \in \mathbb{C}$, we obtain the characteristic equation

$$
m+\gamma e^{-m \tau}=0 .
$$

By setting $m=\alpha+i \beta$, we arrive to the following system of equations:

$$
\begin{gathered}
\alpha+\gamma e^{-\alpha \tau} \cos (\beta \tau)=0 \\
\beta-\gamma e^{-\alpha \tau} \sin (\beta \tau)=0 .
\end{gathered}
$$

Equation (5) reveals that the system admits infinite solutions $(\alpha, \beta)_{k}, k \in \mathbb{Z}$ for each pair of values $\gamma, \tau>0$. Since the DDE is linear, the solutions are given by the general expression:

$$
L(t)=l_{0}+\left(L_{0}-l_{0}\right) \sum_{k=-\infty}^{+\infty} e^{m_{k} t} .
$$

Stable solutions are obtained for $\alpha<0$, and oscillatory solutions whenever $\beta \neq 0$. It can be demonstrated that the following condition holds for oscillatory solutions [18],

$$
\tau \gamma>\frac{1}{e}
$$

while unstable solutions are present whenever

$$
\tau \gamma>\frac{\pi}{2}
$$

These results allow us to define oscillatory and stability limits given by,

$$
\tau_{\text {oscil }}=\frac{1}{e \gamma}, \tau_{s t a b}=\frac{\pi}{2 \gamma}
$$

Also, for $\alpha=0, \beta \tau=\frac{\pi}{2} \pm n \pi$, and thus, at the limit of stability, the solution is oscillatory with frequency $\frac{\pi}{2 \tau}$. Furthermore, for $\beta=0$, we obtain $\alpha=-\gamma e^{-\alpha \tau}<0$, and therefore non-oscillatory solutions are stable. We have solved the DDE in (3) numerically for some values of $\gamma$ and $\tau$. The resulting rest-lengths are shown in Figure 2 and confirm the analytical results. Figure 3 shows the stability diagram, which indicates the regions with oscillatory and stable solutions in the $(\tau, \gamma)$-plane. 


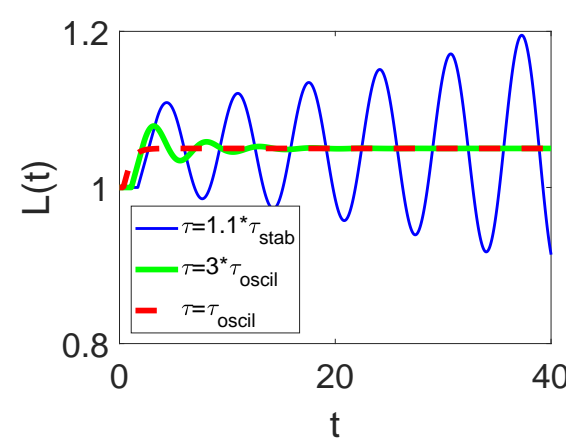

(a)

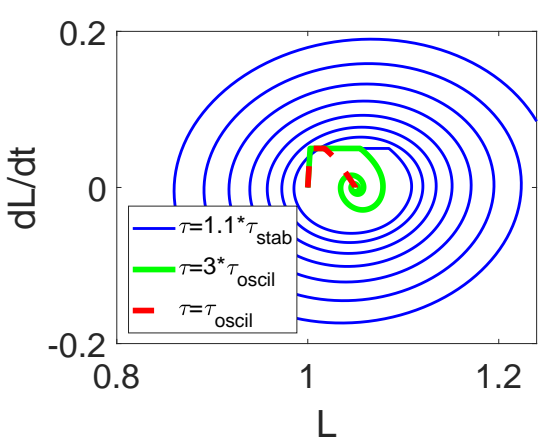

(b)

Figure 2: One element system. (a) Time evolution of rest-length $L(t)$, with one example of non-oscillatory solution (thick dashed red line), a stable oscillatory solution (thick green continuous line), and a unstable oscillatory solution (thin blue continuous line). The three solutions correspond respectively to region I, II and III in Figure 3. (b) Phase diagram of $L(t)$ for the three solutions

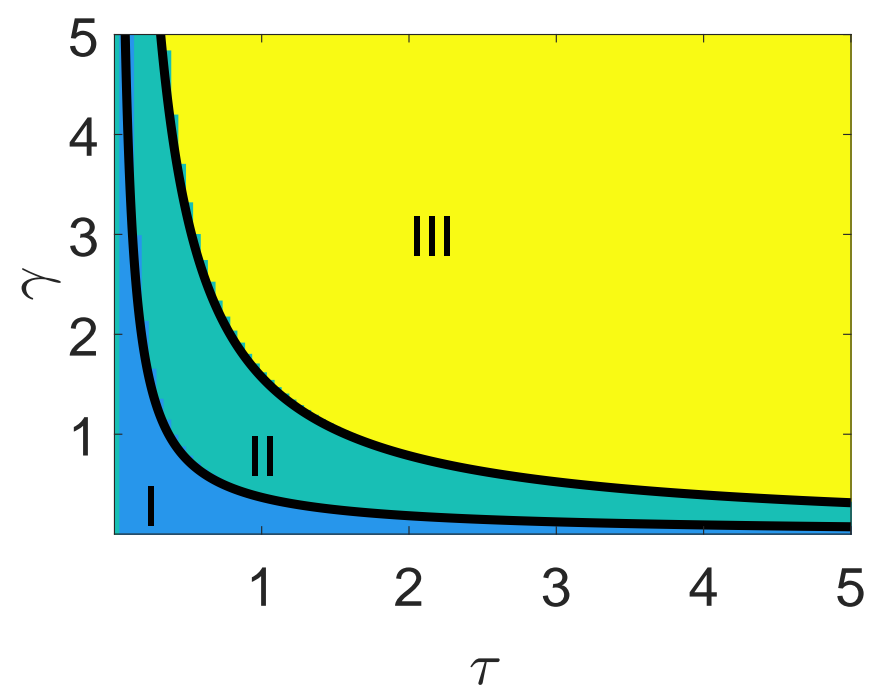

Figure 3: Stability diagram for 1 element. Solutions in region I are nonoscillatory $\left(\tau<\tau_{\text {oscil }}\right)$, in region II are oscillatory and stable $\left(\tau_{\text {oscil }}<\tau<\tau_{\text {stab }}\right)$, while in region III are oscillatory and unstable $\left(\tau_{\text {stab }}<\tau\right)$. Thick lines correspond to functions $\tau \gamma=1 / e$ (boundary regions I and II) and $\tau \gamma=\pi / 2$ (boundary regions II and III). 


\subsection{Two element analysis}

We here analyse the two elements system in Figure 1b. Both ends of the system are fixed and only the node between the elements is allowed to move horizontally with a displacement $u(t)$. The equilibrium condition between the nodes reads $\sigma_{1}=\sigma_{2}$, which for $k_{1}=k_{2}=k$ is equivalent to the kinematic condition,

$$
l_{1}-L_{1}=l_{2}-L_{2} .
$$

with $l_{i}$ and $L_{i}$ the the apparent and rest-length of element $i=1,2$, respectively (see Figure 1b). The time evolution of the rest-lengths of elements 1 and 2 are given by,

$$
\begin{aligned}
& \dot{L}_{1}=\gamma_{1}\left(l_{1}(t-\tau)-L_{1}(t-\tau)\right) \\
& \dot{L}_{2}=\gamma_{2}\left(l_{2}(t-\tau)-L_{2}(t-\tau)\right)
\end{aligned}
$$

After using the fact that $l_{1}+l_{2}=l_{0}=$ const and the stress equilibrium in (9), the previous equations may be expressed as,

$$
\begin{aligned}
& \dot{L}_{1}=\frac{\gamma_{1}}{2}\left(l_{0}-L_{1}\left(t-\tau_{1}\right)-L_{2}\left(t-\tau_{1}\right)\right) \\
& \dot{L}_{2}=\frac{\gamma_{1}}{2}\left(l_{0}-L_{1}\left(t-\tau_{2}\right)-L_{2}\left(t-\tau_{2}\right)\right)
\end{aligned}
$$

This is a set of coupled DDEs. We will analyse its stability and oscillatory response for the simpler case $\gamma_{1}=\gamma_{2}=\gamma$. As before, by suggesting solutions of the type $L_{i}(t)=l_{i}(0)+\left(L_{i}(0)-l_{i}(0) e^{m_{i} t}, i=1,2\right.$, we get similar but coupled stability conditions,

$$
\begin{aligned}
& m_{1}+\frac{\gamma}{2}\left(e^{-m_{1} \tau_{1}}+e^{\left(m_{2}-m_{1}\right) t} e^{-m_{2} \tau_{1}}\right)=0, \\
& m_{2}+\frac{\gamma}{2}\left(e^{-m_{1} \tau_{2}} e^{\left(m_{1}-m_{2}\right) t}+e^{-m_{2} \tau_{2}}\right)=0 .
\end{aligned}
$$

When searching for time-independent values of $m_{i}$, we must have $m_{1}=$ $m_{2}=m$, and thus we arrive to one single equation:

$$
m+\frac{\gamma}{2}\left(e^{-m \tau_{1}}+e^{-m \tau_{2}}\right)=0 .
$$

The oscillatory and stability conditions can be now studied from the system of non-linear equations

$$
\begin{gathered}
\alpha+\frac{\gamma}{2}\left(e^{-\alpha \tau_{1}} \cos \left(\beta \tau_{1}\right)+e^{-\alpha \tau_{2}} \cos \left(\beta \tau_{2}\right)\right)=0, \\
\beta-\frac{\gamma}{2}\left(e^{-\alpha \tau_{1}} \sin \left(\beta \tau_{1}\right)+e^{-\alpha \tau_{2}} \sin \left(\beta \tau_{2}\right)\right)=0 .
\end{gathered}
$$

For non-oscillatory solutions $(\beta=0)$, equation (12) implies that $\alpha<0$, so that non-oscillatory solutions are stable. When $\beta \rightarrow 0$, equations (12)-(13) turn 
into

$$
\begin{aligned}
\alpha+\frac{\gamma}{2}\left(e^{-\alpha \tau_{1}}+e^{-\alpha \tau_{2}}\right) & =0, \\
1-\frac{\gamma}{2}\left(e^{-\alpha \tau_{1}} \tau_{1}+e^{-\alpha \tau_{2}} \tau_{2}\right) & =0 .
\end{aligned}
$$

By setting $p_{1}=\alpha \tau_{1}$ and $p_{2}=\alpha \tau_{2}$, and multiplying (15) by $\alpha$, the oscillatory condition can be found by searching pairs $\left(p_{1}, p_{2}\right)$ that satisfy

$$
e^{-p_{1}}+e^{-p_{2}} \geq-\left(e^{-p_{1}} p_{1}+e^{-p_{2}} p_{2}\right)
$$

and setting

$$
\tau_{i}=\frac{2 p_{i}}{\gamma}\left(e^{-p_{1}}+e^{-p_{2}}\right), i=1,2 .
$$

The stability condition can be determined by setting $\alpha=0$, in which case equations (12)-(13) read

$$
\begin{array}{r}
\cos \left(\beta \tau_{1}\right)+\cos \left(\beta \tau_{2}\right)=0 \\
\beta=\frac{\gamma}{2}\left(\sin \left(\beta \tau_{1}\right)+\sin \left(\beta \tau_{2}\right)\right)
\end{array}
$$

From the first equation we conclude that oscillatory solutions $(\beta \neq 0)$ imply that

$$
\beta \tau_{1}=\beta \tau_{2}+\pi,
$$

which inserted in (19) gives the stability condition:

$$
\frac{\pi}{\tau_{1}+\tau_{2}} \geq \gamma \sin \frac{\pi \tau_{1}}{\tau_{1}+\tau_{2}} .
$$

The stability diagram in Figure 4 indicates the regions with oscillatory and stable solutions for $L_{1}$ and $L_{2}$ for different values of $\gamma$ and in the $\left(\tau_{1}, \tau_{2}\right)$-plane. Due to the kinematic and mechanical coupling, the solution is equally oscillatory or stable for each one of the two elements, and has a larger instability region as $\gamma$ increases.

We also point out that even if the delay of one element satisfies $\tau_{i}>\tau_{s t a b}$, the solution may be stable if the other delay is sufficiently below $\tau_{s t a b}$. Similarly, even if $\tau_{i}>\tau_{\text {oscil }}$, solution may be non-oscillatory when the delay of the adjacent element is below $\tau_{\text {oscil }}$. It can be said, that the delay of one element may help to stabilise or damp the global solution.

The direction along the axis $\tau_{1}=\tau_{2}$ in each stability chart of Figure 4 corresponds to an horizontal line in the stability charts of Figure 3 for the values $\gamma=0.5$ and $\gamma=1$. Indeed, for $\tau_{1}=\tau_{2}$, equations (16) and (20) give the same oscillatory and stability condition for one single element in (8). This can be also deduced from the fact that when the delays in both elements are equal, we have that $\dot{L}_{1}=\dot{L}_{2}$, and the sum of rest-lengths $L=L_{1}+L_{2}$ evolves 


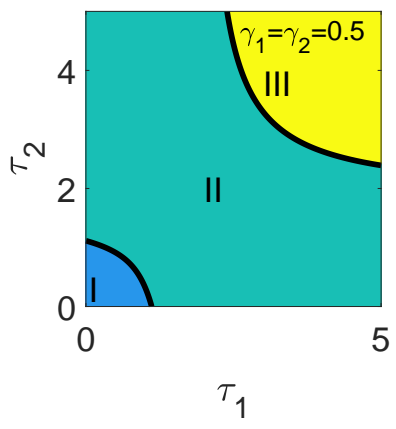

(a)

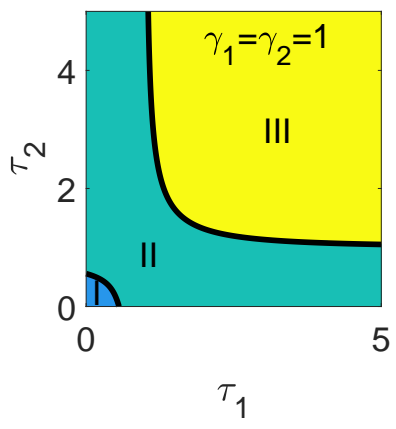

(b)

Figure 4: Stability diagram of 2 element system for different values of $\gamma$ in the $\left(\tau_{1}, \tau_{2}\right)$-plane. Solutions of $L_{1}$ and $L_{2}$ in region I are non-oscillatory $\left(\tau<\tau_{o s c i l}\right)$, in region II are oscillatory and stable $\left(\tau_{\text {oscil }}<\tau<\tau_{\text {stab }}\right)$, while in region III are oscillatory and unstable $\left(\tau_{s t a b}<\tau\right)$. Contour plots have been obtained with numerical solution of DDE, while thick lines correspond to analytical solution in equations (16)-(17) and (20).

as $\dot{L}=\gamma\left(l_{0}-L(t-\tau)\right)$, so that the same critical values of the delay apply for $L_{1}+L_{2}$. For $\tau_{1}=\tau_{2}$ we also have that

$$
\dot{u}=\frac{d}{d t}\left(l_{1}-l_{1}(0)\right)=\frac{1}{2} \frac{d}{d t}\left(l_{0}-\left(L_{1}-L_{2}\right)\right)=0
$$

and thus no oscillations appear in the displacement of the cell boundary. This fact, and the presence of cell area oscillations in experimental measures motivates the use of a size-dependent delay with the form

$$
\tau(t)=\lambda l(t) .
$$

We could not retrieve in this case the analytical critical values of $\lambda$ for stable and oscillatory condition (DDE becomes non-linear). However, we have numerically tested the relation in equation (21), and confirmed that displacement oscillations are obtained for a size-dependent delay, with the same value of $\lambda$ for the two cells, but with unequal initial sizes. The stability diagram in Figure 5 compares the region boundaries of one element (thick lines) and the one using a size-dependent delay. In the latter case, the values of $\tau$ in the horizontal axis correspond to the value of $\lambda$. The initial lengths of each element are equal to $l_{1}(0)=l_{2}(0)=1.0$. For these lengths, the stable region is slightly reduced in the $(\lambda, \gamma)$-plane when compared to the stable region in the $(\tau, \gamma)$-plane.

Figure 6 shows the time evolution of the rest-lengths and displacements for the case with constant but different delay in the two elements (left), and for a size-dependent delay (right). Both give a oscillatory displacement $u(t)$, but for the values of $\tau$ and $\lambda$ tested, the amplitude of the average oscillation diminishes. 


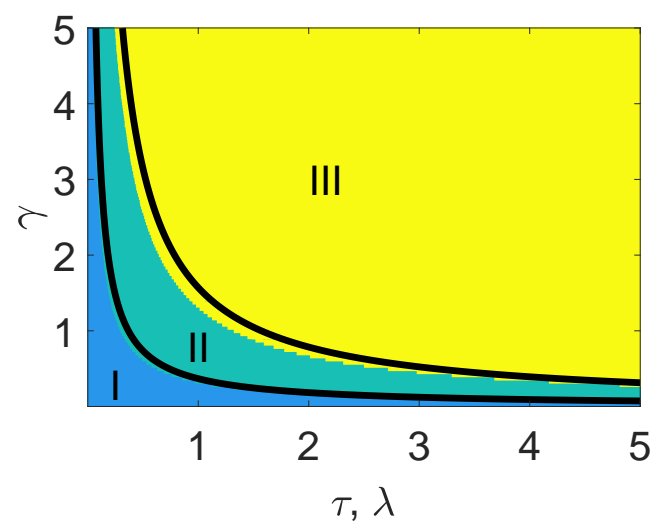

Figure 5: Stability diagram for 2 elements. Thick lines correspond to regions $\tau_{1}=\tau_{2}$ (same lines as in Figure 3). Contour regions correspond to sizedependent delays $\tau_{i}=\lambda l_{i}$, obtained with $l_{i}(0)=1$.



(a)

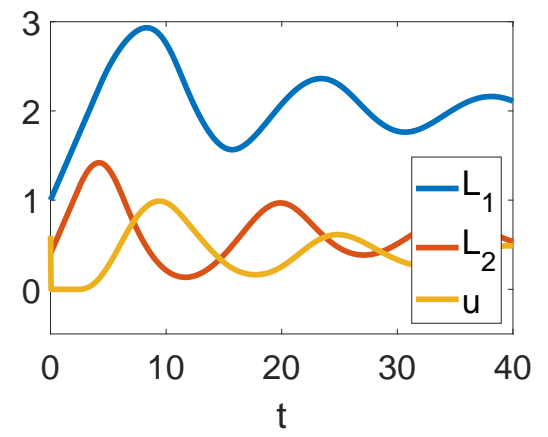

(b)

Figure 6: Time evolution of length $L$ and displacement $u$ for: (a) different delays $\tau_{1}=0.5, \tau_{2}=5$ and (b) size-dependent delays $\tau_{i}=\lambda l_{i}$, with $\lambda=2.5$. The two solutions correspond to the stable oscillatory regions (II) in Figure 5.

We note that the use of a size-dependent delay is motivated not only by the triggering of oscillations at the cell boundaries, but also by potential physical hypotheses. For instance, 1) biochemical signalling between the nucleus and the cortex may take longer on larger cells, 2) the effects of (de)polymerisation of actin may take longer to build up, or 3) a globally contractile cortex requires more time to form on larger domains. None of these explanations has been confirmed, but are possible explanations for justifying the more realistic response of a size-dependent delay. 


\section{Application to vertex multicellular model}

In order to reproduce the observed oscillations of cell areas, we have applied the delayed evolution law in (2) to a vertex model containing 16 cells (see Figure $7 a)$. Vertex elements at the cell boundaries have a variable rest-length. Like in the two element system, mechanical equilibrium is imposed at each cell centre $\boldsymbol{x}^{i}$ (nodes) and at each cell cell boundary point $\boldsymbol{y}^{I}$ (vertices). The balance equations of the vertices are transported to the cell centres, in order to have the latter as the sole degrees of freedom of the system. The resulting discrete equations are equivalent to minimising the following elastic potential [21],

$$
\begin{aligned}
\Psi(\boldsymbol{x})= & \sum_{i j} k_{x}\left(l\left(\boldsymbol{x}^{i}-\boldsymbol{x}^{j}\right)-L^{i j}\right)^{2} \\
& +\sum_{I J} k_{y}\left(l\left(\boldsymbol{y}^{I}(\boldsymbol{x})-\boldsymbol{y}^{J}(\boldsymbol{x})\right)-L^{I J}\right)^{2}
\end{aligned}
$$

where $L^{i j}$ and $L^{I J}$ are respectively the rest-lengths of the nodal element $i j$ and the vertex element $I J$, and $l(\boldsymbol{v})$ denotes the length of vector $\boldsymbol{v}$. Material parameter $k_{x}$ is the stiffness of the nodal network joining the cell centres, while $k_{y}$ is the stiffness of the network joining the vertices. The positions of the vertices $\boldsymbol{y}^{I}$ is computed by imposing their location at the barycentres of the nodal triangulation defined by $\boldsymbol{x}^{i}$. In addition to the elastic potential given in (22), a quadratic term penalising area variations is added, so that cell area preservation is numerically simulated, although not exactly imposed.



(a)

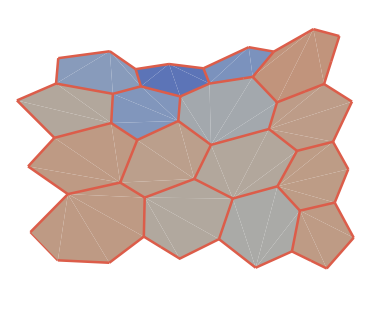

(b)

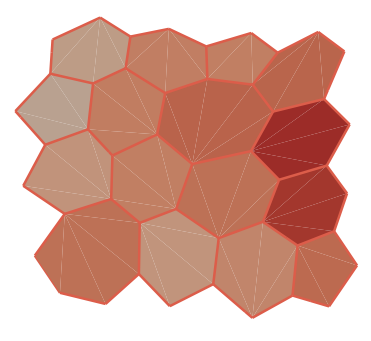

(c)

Figure 7: Undeformed and deformed cells for the vertex model at time $\mathrm{t}=15 \mathrm{~min}$. (a) Undeformned cells. (b) Deformed cells at $t=15$ using constant delay $\tau_{1}=\tau_{2}=1.1$. (c) Deformed cells at $t=15$ using a size-dependent delay $\tau_{i}=1.65 l_{i}, i=1, \ldots, N_{\text {cells }}$. Cell colours denote relative area changes with respect to initial configuration in (a).

A delay on the rest length evolution of the vertex elements has been applied, 
while the rest-lengths of the nodal elements are kept constant. The model uses a network of cell centres, which is fixed on its left and right ends.

We have numerically verified the presence of oscillatory and stability limits of the vertex displacements as a function of the delay. We have tested the effects of having a constant and a size-dependent delay. In the former case, all the cells use the same delay, which results in synchronised oscillations for all the cells and a periodic evolution of each area. In the latter case instead, oscillations are progressively desynchronised, giving rise to a more realistic dynamics, that is, with a total area that has lower variations (see thick line in Figure 8). This fact prompts us to also support the presence of a size-dependent delay. This may be associated to the signalling process from the nuclei to the cortex or (de) polymerisation process and turn over of molecules between the cortex and the cytoplasm. As yet though, further experimental evidence is necessary to confirm these explanations.

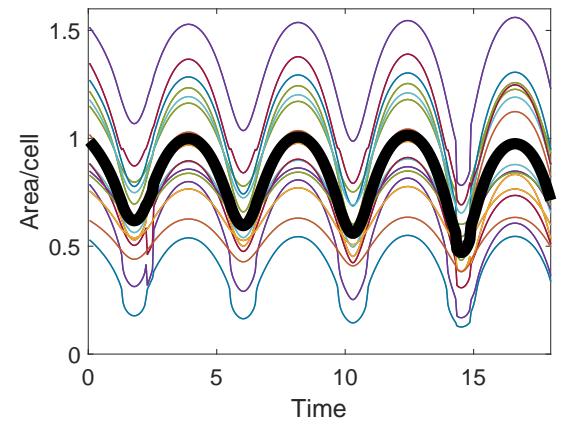

(a)

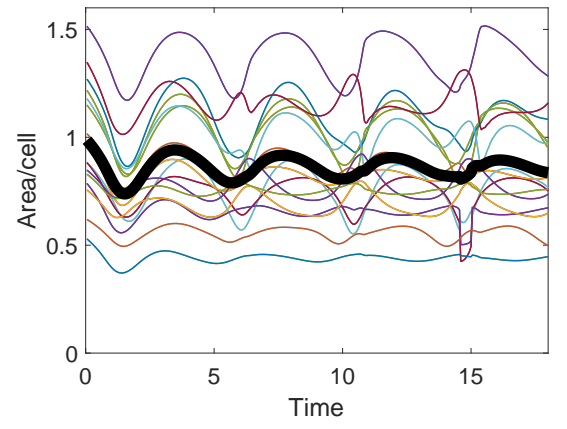

(b)

Figure 8: Time evolution of each cell area $A_{i}$ (thin lines) and mean area $\bar{A}$ (thick line) of a $4 \times 4$ monolayer shown in Figure 7. (a) Constant delay $\tau_{1}=\tau_{2}=1.1$. (b) Size-dependent delay $\tau_{i}=1.65 l_{i}, i=1, \ldots, N_{\text {cells }}$.

Figure 9 shows the stability diagram obtained from the numerical simulation. The regions resemble the two-element system, but due to the non-linearity of the problem, the boundaries of the regions are less clear. The jagged boundary between the regions may be attributed to the solution of the non-linear equations with a backward Euler scheme and Newton-Raphson algorithm, which requires the use of tolerances for its convergence, and also to the numerical evaluation of the stability from the initial 200 increments. In some cases, this time interval may not be sufficient for a complete characterisation of the dynamics.

It can be inferred from Figure 9 that region II, with stable oscillations, is reduced when a constant delay is used, while unstable oscillations are obtained for a wider range of values. Interestingly, in the case of a size-dependent delay, the region of unstable oscillations is reduced for the vertex system. Although we do not have a direct explanation for this, it is likely that the interaction and desynchronisation of the oscillations helps to stabilise the cell deformations. 


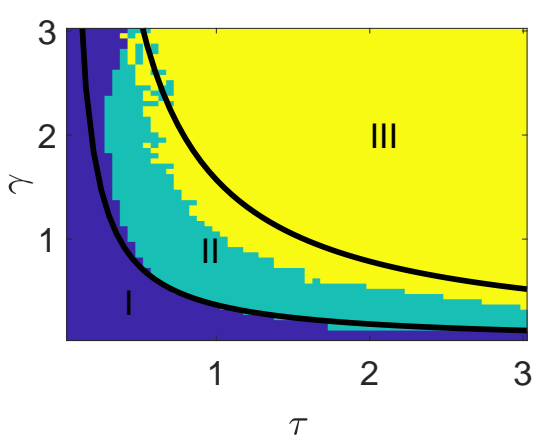

(a)

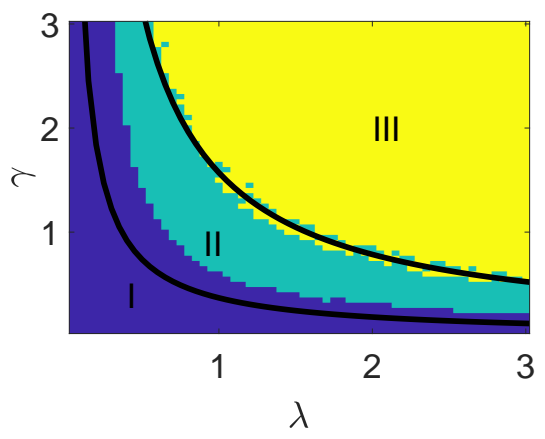

(b)

Figure 9: Stability diagram of vertex model obtained through numerical simulations. (a) Diagram with constant delay in the $(\tau, \gamma)$. Comparison with analytical results for 1 element (thick lines). (b) Size-dependent delay, $\tau_{i}=\lambda l_{i}$. Numerical results evaluated from first first 200 time increments of simulation. Region I: non-oscillatory response, region II: oscillatory stable response, region III: oscillatory unstable response. Thick lines correspond to the one-element analytical boundaries, which are plotted as a reference.

\section{Conclusions}

We have hypothesised a size-dependent delay, which is sufficient to exhibit displacement oscillations at the cell boundaries. The model does not resort to diffusion-reaction equations, but requires either elements with different delays or a size-dependent delay. We show that such simplified model is able to induce oscillatory behaviour and resonance-like response, even in the absence of applied forces, reaction terms or external sources.

The model couples mechanics and a delay differential equation that determines the evolution law of the rest-length. Such rest-length changes have been used in embryonic tissue [22, 23], but had not previously been employed in the analysis of oscillatory behaviour. We here link the presence of the oscillation and compute the critical delays that may trigger the oscillations or instabilities like those observed during embryogenesis.

Rest-length changes have been successfully employed in embryogenesis [22, 23], and experimentally measured and calibrated [26]. We here further extend these models to also explain the observed oscillations by integrating the delay between mechanical and chemical signalling [10]. In summary, the model presented here bridges simple cell rheology with periodic responses, and explicitly quantifies its correlation with observed strain oscillations.

Although we do not analyse the causes of the delay, we make a direct link between this delay and the mechanical response of multicellular systems. It has already been pointed out that the use of coupling delays can induce a rich variety of behaviours [27]. We in fact show that even simple DDEs, coupled 
with mechanical equilibrium, are able to produce wave-like oscillations. Further quantification of the estimated delay and rest-length changes is still necessary, while application of the methodology to three-dimensional tissues, giving rise to out-of-plane deformations is currently under study. Since cells have different dimensions along lateral or apical directions, a size-dependent delay would imply different oscillations or instabilities in in-plane and out-of-plane deformations, giving rise to the emergence of bending or cell intercalations. Three-dimensional equilibrium conditions may though attenuate such instabilities, which demands further numerical simulations for a in depth analysis. Such study is currently under investigation.

Oscillatory deformations in tissues are ubiquitous and have been measured thorough area fluctuations $[9,10,17]$. The analysis described here should not be discarded as a cause of these deformations or of the instabilities observed in embryogenesis.

\section{Acknowledgements}

JJM is financially supported by the Spanish Ministry of Economy and Competitiveness (grant DPI2016-74929-R) and by Generalitat de Catalunya (grant 2017 SGR 1278).

\section{References}

[1] S. Chakraborty, M.H. Jensen, S. Krishna, B. M. Pers, S. Pigolotti, V. Sekara, and S. Semsey. Limit-cycle oscillations and stable patterns in repressor lattices. Phys. Rev. E, 86:031905, 2012.

[2] W.J. Rappel, F. Fenton, and A. Karma. Spatiotemporal control of wave instabilities in cardiac tissue. Phys. Rev. Letters, 83:456, 1999.

[3] S. Puwal and B.J. Roth. Monodomain shear wave propagation and bidomain shear wave dispersion in an elastic model of cardiac tissue. Phys. Rev. E, 87:024701, 2013.

[4] O.V. Popovych, S. Yanchuk, and P.A. Tass. Delay- and coupling-induced firing patterns in oscillatory neural loops. Phys. Rev. Letters, 107:228102, 2011.

[5] S. Ares, L.G. Morelli, D.J. Jörg, A.C. Oates, and F. Jülicher. Collective modes of coupled phase oscillators with delayed coupling. Phys. Rev. Letters, 108:204101, 2012.

[6] A.C. Oates, L.G. Morelli, and S. Ares. Patterning embryos with oscillations: structure, function and dynamics of the vertebrate segmentation clock. Development, 139:625-639, 2012. 
[7] J. D. Murray. Mathematical Biology. Springer, New York, 2nd edition, 1993.

[8] L. Zhang, S. Zhang, H. Tong, D. Lei, and B. Hu. Spontaneously periodic wave generation in coupled excitable media. Phys. Rev. E, 79:056213, 2009.

[9] J Solon, A Kaya-Copur, and D Brunner. Pulsed forces timed by a ratchetlike mechanism drive directed tissue movement during dorsal closure. Cell, 58(137):1331-1342, 2009.

[10] K. Dierkes, A. Sumi, J. Solon, and G. Salbreux. Spontaneous Oscillations of Elastic Contractile Materials with Turnover. Phys. Rev. Letters, 113:148102, 2014.

[11] P. Rodriguez-Franco, A. Brugués, A. Marin-Llaurado, V. Conte, G. Solanas, E. Batlle, J.J. Fredberg, P. Roca-Cusachs, R. Sunyer, and $\mathrm{X}$. Trepat. Long-lived force patterns and deformation waves at repulsive epithelial boundaries. Num. Math., 16(10):1029-1037, 2017.

[12] M. Rauzi, U. Krzic, T.E. Saunders, M. Krajnc, P. Ziherl, L. Hufnage, and M. Leptin. Embryo-scale tissue mechanics during Drosophila gastrulation movements. Nat. Commun., 6:8677, 2015.

[13] AC Martin, M Kaschube, and EF Wieschaus. Pulsed contractions of an actin-myosin network drive apical constriction. Nature, 457:495-499, 2009.

[14] R. Levayer and T. Lecuit. Oscillation and polarity of e-cadherin asymmetries control actomyosin flow patterns during morphogenesis. Dev Cell. 26(2):162-75, 2013.

[15] J.B. Michaux, F.B. Robin, W.M. McFadden, and E.M. Munro. Excitable RhoA dynamics drive pulsed contractions in the early $C$. elegans embryo. J. Cell Biol.. In press. doi.org/10.1083/JCB.201806161.

[16] M. Roh-Johnson, G. Shemer, C.D. Higgins, J.H. McClellan, A.D. Werts, U.S. Tulu, L. Gao, E. Betzig, D.P. Kiehart, and B. Goldstein. Triggering a cell shape change by exploiting preexisting actomyosin contractions. Science, 335:1232-1235, 2012.

[17] J.L. Teo and A.S. Yap. Cycling rho for tissue contraction. J. Cell Biol., 214(5):495-498, 2016.

[18] T. Erneux. Applied Delay Differential Equations, volume 3 of Surveys and Tutorials in the Applied Mathematical Sciences. Springer, New York, 2009.

[19] J. Sedzinski, M. Biro, A. Oswald, J.Y. Tinevez, G. Salbreux, and E. Paluch. Polar actomyosin contractility destabilizes the position of the cytokinetic furrow. Nature, 476:462-463, 2011. 
[20] J.J. Muñoz, V. Conte, N. Asadipour, and M. Miodownik. A truss element for modelling reversible softening in living tissues. Mech. Res. Comm., 49:44-49, 2013.

[21] P. Mosaffa, A. Rodríguez-Ferran, and J.J. Muñoz. Hybrid cellcentred/vertex model for multicellular systems with equilibrium-preserving remodelling. Int. J. Num. Meth. Biomed. Engng., 34(3):1-24, 2018.

[22] R. Clément, C. Collinet, B. Dehapiot, T. Lecuit, and P.F. Lenne. Viscoelastic dissipation stabilizes cell shape changes during tissue morphogenesis. Biorxiv, 2017. doi.org/10.1101/107557.

[23] K. Doubrovinski, M. Swan, O. Polyakov, and E.F. Wieschaus. Measurement of cortical elasticity in drosophila melanogaster embryos using ferrofluids. Proc. Natl. Acad. Sci. USA, 114(5):1051-1056, 2017.

[24] G. Stépán. Retarded dynamical systems: stability and characteristic functions. Longman Scientific \& Technical, 1989.

[25] C.E. Falbo. Analytic and numerical solutions to the delay differential equations. In Joint Meeting of the Northern and Southern California Sections of the MAA, San Luis Obispo, CA, 1995.

[26] N. Khalilgharibi, J. Fouchard, N. Asadipour, R Barrientos, M Duda, A. Yonis, A. Harris, P. Mosaffa, Y. Fujita, A. Kabla, Y. Mao, B. Baum, J.J. Muñoz, M. Miodownik, and G. Charras. Stress relaxation in epithelial monolayers is ocntrolled by actomyosin cortex. BioRxiv, 2018. doi.org/10.1101/302158

[27] K. S. Yeung and S. H. Strogatz. Time delay in the kuramoto model of coupled oscillators. Phys. Rev. Letters, 82:648-268, 1999. 